\title{
Lidil
}

Revue de linguistique et de didactique des langues

\section{Le brouillon en FLE : quelle importance lui accordent les enseignants du secondaire en Algérie?}

Souad Benabbes

\section{OpenEdition}

Journals

Édition électronique

URL : http://journals.openedition.org/lidil/4003

DOI : $10.4000 /$ lidil.4003

ISSN : 1960-6052

Éditeur

UGA Éditions/Université Grenoble Alpes

Édition imprimée

Date de publication : 30 mai 2016

Pagination : 185-205

ISBN : 978-2-84310-326-1

ISSN : 1146-6480

Référence électronique

Souad Benabbes, « Le brouillon en FLE : quelle importance lui accordent les enseignants du secondaire en Algérie? », Lidil [En ligne], 53 | 2016, mis en ligne le 01 janvier 2017, consulté le 30 octobre 2020. URL : http://journals.openedition.org/lidil/4003 ; DOI : https://doi.org/10.4000/lidil.4003 


\title{
Le brouillon en FLE : quelle importance lui accordent les enseignants du secondaire en Algérie?
}

\author{
Souad Benabbes*
}

\begin{abstract}
RÉSUMÉ
Suite à un état des lieux de la réflexion didactique sur les fonctions du brouillon en classe, cet article présente et analyse les résultats d'une enquête menée auprès d'enseignants algériens du secondaire et portant sur la place qu'ils accordent à cet écrit dans leur enseignement de la production écrite, et plus largement sur les représentations qu'ils se font de cet outil. Via un questionnaire, les 34 enseignants interrogés s'expriment également sur les difficultés que rencontrent leurs élèves en situation de production écrite et sur leurs pratiques pédagogiques, y compris la manière dont ils évaluent les écrits de leurs élèves.
\end{abstract}

\begin{abstract}
Following an overview of the didactic reflections on the functions of drafts in teaching writing process, this article presents and analyzes the results of a survey of Algerian school teachers concerning the place they give to the draft in writing teaching. They are more broadly interviewed about their representations of the place and use of drafts. Via a questionnaire, 34 teachers surveyed also expressed about the difficulties of their students regarding written production and about their teaching practices, including how they evaluate the writings of their students.
\end{abstract}

\section{Introduction}

Chacun de nous a déjà écrit un brouillon dans sa vie scolaire, qui, une fois le texte final produit, a été déposé dans une corbeille. La vie éphémère du brouillon est liée à son statut d'écrit préliminaire qui s'efface

* Université Hadj Lakhder, Batna. 
devant la version finale de l'écrit réalisé. Ce sont précisément ces écrits destinés à ne pas être lus par l'enseignant qui peuvent être source d'un aménagement didactique susceptible de faciliter et d'améliorer l'écriture des jeunes scripteurs. En focalisant l'attention sur les brouillons des élèves, on s'intéresse à une phase transitoire de la production écrite qui se manifeste à travers les hésitations et les tâtonnements de l'écriture. Cette étape semble d'autant plus intéressante à analyser qu'elle est souvent cachée, négligée et perçue comme inutile. Or, elle constitue une étape préparatoire à la diffusion d'un texte, nécessaire à la production d'un écrit de qualité.

En effet, «[...] écrire et réécrire sont compris comme deux faces d'une même activité, la seconde apparait lorsque le scripteur, par lecture-révision perçoit des dissonances entre son projet textuel et le texte déjà produit» (Cuq, 2003, p. 112). C'est pourquoi le pré-rédactionnel et la réécriture apparaissent actuellement comme des éléments constitutifs de l'activité d'écriture et de son apprentissage dont les enseignants doivent tenir compte. De plus en plus, on mesure les limites d'un travail correctif sur le produit final pour accorder toute son importance au processus rédactionnel.

À l'école algérienne, les élèves sont souvent confrontés à la rédaction de différents types de texte, pour laquelle ils doivent mobiliser et développer des savoirs, des savoir-faire et des savoir-être, en alternant travaux individuels et travaux en groupes de pairs. Les concepteurs des guides pédagogiques du manuel de $3^{\mathrm{e}}$ année secondaire, destinés aux enseignants du français, insistent sur la mise en œuvre d'ateliers d'écriture où le travail effectué, du brouillon au texte final, ne s'accomplit pas d'un seul mouvement, mais en séances successives ou interrompues par des pauses d'écriture et des retours sur le déjà-écrit, faisant ainsi du premier jet un objet mobile, évaluable, qui prépare au texte final. Le scripteur ne peut donc pas aboutir au produit final sans passer par la transformation, le réaménagement de son texte. Selon les concepteurs du guide, dont l'extrait ci-dessous est issu, la «mise en chantier du projet d'écriture» doit passer par les étapes suivantes :

- rédaction du premier jet : chaque apprenant individuellement ou en groupes restreints rédige une première version;

- mise en texte : il s'agit maintenant de socialiser ses premiers écrits et les soumettre à ses pairs dont les remarques et observations aideront à prendre conscience des effets de lecture produits par chaque texte; 
- évaluation du premier jet : elle revêt la forme d'une confrontation du texte à la liste des critères pour guider l'amélioration du premier jet;

- finalisation : elle se caractérise sous la forme d'une réécriture ou de plusieurs réécritures successives, avec retour au premier jet, avant la rédaction finale. (MEN, 2006, p. 31-32)

Pourtant, malgré ces préconisations, nombreux sont les élèves qui arrivent au lycée sans vraiment savoir faire un brouillon, sans même en voir l'utilité. Pour eux, faire un brouillon, c'est gaspiller du temps. Selon Reuter (2002), les apprenants considèrent le brouillon comme un signe d'incompétence : ils ont tendance à l'associer à quelque chose de sale, d'illisible, empli de taches et de ratures. Une des raisons évoquées par Reuter à cette représentation négative est liée au fait que les élèves sont habitués à lire et à analyser des textes dans leur version définitive, propres et exempts de toute trace de leur fabrication. Le peu de cas souvent accordé au brouillon par les élèves explique sans doute l'effet provoqué par leurs écrits qui, à première vue, peuvent donner l'impression de ne pas être achevés, de n'être qu'un premier jet raturé et désorganisé qui nécessiterait de multiples corrections. Cela dit, si le brouillon est souvent déprécié du scripteur, il est également peu considéré par les enseignants.

Notre expérience, appuyée par des échanges informels avec de nombreux collègues, nous a permis de faire l'hypothèse que les enseignants algériens n'attirent pas souvent l'attention de leurs élèves sur la nécessité de faire un brouillon; faute de temps, ils les amènent parfois à se précipiter dans leur texte. En outre, la volonté de l'enseignant, que l'on observe parfois, de faire rédiger ses élèves le plus vite possible au «propre» peut cacher une volonté de fuir le brouillon, voire l'activité d'écriture. Lors de l'évaluation des écrits scolaires, les brouillons ne sont pas pris en considération par de nombreux enseignants qui appellent leurs élèves à achever impérativement leur texte définitif. Pour l'institution scolaire, le brouillon n'est pas un objet destiné à être corrigé; seul le produit final est mis en valeur et devrait être évalué par les enseignants. Cette représentation réductrice du brouillon peut constituer un obstacle à la construction d'une conception de l'écriture comme travail en plusieurs jets, nécessitant des tâches de réécriture.

Il est donc nécessaire de promouvoir l'utilité du brouillon dans le processus d'écriture en classe en vue d'amener les apprenants à écrire plusieurs versions permettant d'aboutir à un texte définitif de qualité. Le brouillon devrait permettre aux scripteurs d'amoindrir la charge 
cognitive impliquée par l'activité d'écriture, comme l'explique Penloup (1994) : «Faire un brouillon permet de ne pas affronter toutes les difficultés à la fois en hiérarchisant les tâches, en négligeant délibérément, dans un premier temps, les problèmes de surface.» (p. 30) On voit ainsi l'utilité de légitimer la place et l'intérêt de l'activité de réécriture au regard des élèves par l'utilisation judicieuse du brouillon comme outil dans le cursus d'enseignement/apprentissage de l'écrit. Cela dit, qu'en pensent les enseignants? Notre questionnement de recherche est double : quelle est la place accordée au brouillon dans l'enseignement/apprentissage de la production écrite en FLE par les enseignants? Le brouillon fait-il l'objet d'une évaluation dans les pratiques enseignantes?

Pour répondre à ces deux questions, nous nous fonderons sur les résultats partiels d'une enquête par questionnaire auprès de 34 enseignants de français. Mais auparavant, nous reviendrons sur l'apport de la génétique textuelle dans la didactisation du brouillon scolaire en présentant les principaux travaux qui ont été faits à ce sujet.

\section{Cadre théorique}

\subsection{Production écrite et psychologie cognitive}

Les recherches consacrées à la production écrite ont été centrées majoritairement sur l'analyse du texte final, résultat du processus rédactionnel. Des études menées depuis 1980 mettent l'accent sur les processus cognitifs mis en œuvre dans la production écrite. Les approches cognitives cherchent à recenser tous les actes et processus impliqués dans l'activité d'écriture. Comme le note Carter (1990), le foisonnement de ces travaux sur le processus rédactionnel se justifie par «l'intérêt croissant pour les processus cognitifs en psychologie et en psycholinguistique» (p. 115).

Dans ce domaine, les travaux des équipes américaines, notamment ceux initiés par Flower et Hayes (1980), constituent un important courant qui a fortement marqué les travaux didactiques. Élaboré il y a presque quarante ans, le premier modèle de Hayes et Flower a été largement dominant et diffusé en France, particulièrement auprès des enseignants, en vue de leur offrir des conceptions des différents processus mis en œuvre dans la production écrite. Hayes et Flower conçoivent la production écrite comme «une situation problème» et ont élaboré un modèle selon lequel trois composantes sont impliquées dans l'activité d'écriture : l'environnement de la tâche, la mémoire à long terme 
et les processus d'écriture proprement dits. La première concerne le contexte dans lequel l'activité se déroule. L'environnement de la tâche inclut tous les éléments extérieurs pouvant affecter le déroulement de la production (la consigne proposée, le thème, les attentes du lecteur); la mémoire à long terme comprend toutes les connaissances relatives au thème, $\mathrm{au}(\mathrm{x})$ destinataire(s) et aux processus d'écriture; les processus rédactionnels proprement dits sont constitués de la planification, de la mise en texte et de la révision.

\subsection{De la génétique textuelle à la didactique du pré-rédactionnel}

Depuis les années 1970, les recherches en génétique textuelle ont particulièrement insisté sur l'enjeu et l'intérêt d'approcher l' «avant-texte». Les brouillons d'écrivains sont devenus des objets d'étude privilégiés. Témoins des lieux de gestation de l'œuvre par excellence, les traces de tâtonnements, les «échecs rattrapés », la recherche du «meilleur état du texte» font voir ce dernier comme un «chantier» (Bellemin-Noël, 1977, p. 5). Ils donnent un aperçu des intentions des écrivains et offrent ainsi aux lecteurs une opportunité exclusive de découvrir ce que signifie l'activité d'écriture pour un écrivain. La didactique gagne à s'inspirer de ces recherches, c'est ce que propose Fabre-Cols (2002) en défendant une approche fine des brouillons de jeunes scripteurs :

[...] en cessant de les croire simples et sans intérêt, en devenant curieux des problèmes qui s'y inscrivent, des systèmes en construction qui s'y posent, en donnant toute leur force aux indices matériels de chaque page, aux hypothèses qui pourraient sous-tendre les erreurs, aux ratures et aux abandons, aux conditions énonciatives de production, aux représentations et aux projets qui animent le sujet-scripteur, à son désir de dire qui bouscule toujours un peu le savoir-écrire... ainsi qu'on le ferait pour n'importe quel texte. (p. 14)

Le brouillon est en effet le premier support qui lève le voile sur l'élaboration d'un produit fini permettant à l'enseignant d'évaluer le degré de développement de la compétence scripturale de l'apprenant et son investissement dans la reconstruction de ses connaissances. Lorsque l'élève écrit un premier jet, l'usage veut que l'enseignant le lise, l'annote et lui retourne ses commentaires, remarques et éventuelles rétroactions écrites. Il convient d'expliciter ce que signifie la rétroaction écrite. 


\subsection{La rétroaction écrite}

Dans un contexte scolaire, la rétroaction consiste en un dialogue entre l'enseignant et les apprenants, elle concerne l'information qu'un élève reçoit à propos d'une action, de ses forces et de ses faiblesses, de l'exactitude d'une réponse et peut lui suggérer des pistes de correction en vue d'améliorer sa performance et son apprentissage (Brown, 2007). Doré (1981) avance qu' «un des moyens dont dispose l'enseignant pour aider l'apprenant à réaliser un apprentissage est de lui fournir une information portant sur l'adéquation ou l'inadéquation de sa réponse par rapport à une performance attendue ( (cité par Hassan, 2011, p. 43).

Selon Rodet (2000), la correction écrite permet à l'évaluateur d'avoir le temps nécessaire à la construction de ses propos et à l'élève de lire plusieurs fois les commentaires. Ainsi, l'apprenant peut réaliser un véritable travail cognitif et métacognitif. La rétroaction permet non seulement de faire progresser l'expression écrite des élèves, mais aussi d'augmenter leur confiance en eux lorsqu'ils sont en situation de rédaction. Les élèves se sentent impliqués dans l'amélioration de leur production écrite lorsqu'ils ont la possibilité de choisir ou non de tenir compte des rétroactions lors des révisions. Les élèves doivent toujours se sentir libres d'appliquer les commentaires à leur gré. Ainsi, la rétroaction doit apparaitre suggestive plutôt que normative. Selon Peterson (2010) :

Afin de favoriser l'appropriation des travaux de production écrite par les élèves, la rétroaction doit :

- démontrer aux élèves les effets positifs de leur texte sur le lecteur;

- identifier les éléments devant éventuellement être retravaillés par les élèves afin de clarifier le sens du texte ou de mieux accrocher le lecteur;

- prendre la forme de suggestions, d'observations et de questions ouvertes plutôt que d'instructions et de critiques. (p. 3)

\subsection{Ce que nous apprennent les recherches sur la pratique du brouillon}

La première recherche de grande ampleur sur les brouillons d'écoliers (en l'occurrence de CM2), menée par Fabre en 1987, a porté sur des textes rédigés selon trois variables de passation : l'une avec écriture, relecture et copie d'un seul trait, l'autre avec relecture et copie le lendemain de la rédaction du premier brouillon, la troisième avec relecture et copie une semaine après la rédaction initiale. La recherche tente 
de repérer les différentes modalités de l'activité métalinguistique des jeunes scripteurs dans le processus d'écriture en fonction de leur âge et de leur niveau. Cette étude (Fabre, 1987) peut être considérée comme la première à établir un lien entre les théories génétiques littéraires et les processus d'écriture des apprentis scripteurs.

Une autre recherche menée par Piolat, Roussey et Fleury (1994) s'est intéressée aux pratiques du brouillon chez les étudiants et à leur impact sur la qualité de la copie. Ils distinguent trois types : les premiers, nommés «jet de notes» comportent des mots, des bribes de phrases, sans qu'un texte ou partie de texte ait été formulé; les deuxièmes sont caractérisés par des éléments textuels mis en relation et hiérarchisés et les derniers montrent une rédaction de phrases entières déjà assemblées pour faire un texte.

Les résultats montrent que les scripteurs qui ont produit un brouillon du deuxième type sont les mieux notés. Ce type de brouillon est caractérisé par une mise en ordre chronologique des idées, prêtes à être linéarisées lors des opérations de mise en texte. Les scripteurs ayant utilisé un brouillon du premier type («jet de notes») obtiennent les notes les plus faibles. Les auteurs expliquent ce résultat par le fait que «brouillonner ainsi traduirait une difficulté à déclencher une stratégie comme celles des Connaissances Racontées ou Transformées » (Piolat, Roussey \& Fleury, 1994, p. 47). Ces résultats montrent que l'activité du scripteur lors du brouillon exerce une influence quant à la qualité du texte produit postérieurement.

Là où les auteurs précédents distinguent fondamentalement trois types de brouillons, Alcorta (2001) n'en retient que deux : les brouillons présentés sous forme de listes, de plans, etc., et les brouillons rédigés. Selon elle, le brouillon «n'est pas la simple projection graphique de quelque chose qui est déjà là, "prêt dans la tête", mais [il] participe de l'extérieur, à la construction de l'architecture mentale du scripteur» (p. 98). Dans cette perspective, elle attribue au brouillon un statut d'outil de complexification de l'activité d'écriture. Elle constate que la nature et la fonction du brouillon subissent des évolutions et des réorientations au cours de la scolarisation des élèves du primaire à l'université et distingue deux sortes de brouillons agissant à différents niveaux du processus d'écriture :

a. Un brouillon linéaire qui ressemble au premier jet du produit final où scripteur et lecteur se confondent. Il est rédigé sous forme de phrases développées et présente peu de différences avec le texte final. De ce fait, les jeunes scripteurs «planifient, récupèrent les 
informations en mémoire et rédigent simultanément» (p. 100). Le but du brouillon linéaire est d'améliorer la communication en agissant sur les opérations de linéarisation. Sa véritable fonction est de servir de base à la production finale. Il offre un retour réflexif sur l'écrit puisque le scripteur peut dans ce cas relire et réécrire le texte entier ou seulement certaines parties;

b. Un brouillon instrumental qui se distingue du brouillon linéaire par ses «structures écrites qui rompent avec l'aspect linéaire de l'écrit de communication» (p. 98). La fonctionnalité de ce brouillon est tout autre. Il ne s'agit plus de disposer d'un écrit complet, mais de «représentations graphiques externes qui servent d'appui à la gestion interne du texte» (p. 100). Le brouillon forme ainsi un appui à la planification, à la hiérarchisation et à l'organisation des informations. C'est un écrit où le scripteur se parle à lui-même, où il peut à la fois mémoriser, planifier, construire et contrôler.

Lors de cette recherche sur les aspects développementaux de l'écriture, l'auteure constate que les brouillons linéaires et instrumentaux n'ont pas la même fonction, car ils n'agissent pas au même niveau du processus rédactionnel. Au fur et à mesure que le niveau scolaire et le degré de maitrise de la pratique scripturale augmentent, le recours au brouillon instrumental se développe alors que le brouillon linéaire est délaissé. Les scripteurs les plus jeunes rédigent leurs textes en utilisant la syntaxe du langage écrit avec des phrases développées. Vers la fin du collège, on constate que la structure de phrases complètes disparait au profit de nouvelles formes d'écrit qui réduisent l'écrit.

Plus proche de notre terrain algérien, une recherche menée par Dembri (2008) cherche à évaluer l'impact de l'usage du brouillon sur l'acquisition du savoir-écrire à l'école. Son étude concerne des élèves de $3^{\mathrm{e}}$ année secondaire. Les élèves ont tout d'abord lu différents textes d'un même genre textuel (l'argumentation), puis ils ont été appelés à réinvestir leurs lectures dans l'écriture d'un texte argumentatif, portant sur un thème différent. Cette phase d'écriture a été suivie de plusieurs réécritures à partir de leurs brouillons. Le but de cette recherche était de comparer les différentes productions des apprenants, de la première à la dernière version, tout en analysant la façon dont ces derniers révisent et modifient leur texte en fonction du guidage qui leur a été fourni.

Dembri (2008) affirme que la qualité des versions finales «est [...] en relation directe avec la pratique du brouillon et la réécriture» (p. 203). 
Mais il faut en persuader les apprenants et les enseignants car au départ, ni les premiers ni les seconds n'en sont convaincus. Ainsi, «introduire le brouillon et la réécriture dans la production de l'écrit nécessite [...] une phase de négociation avec les apprenants pour qu'ils comprennent l'utilité de l'usage du brouillon comme outil qui permet d'avoir un produit fini de qualité, du fait de la réécriture» (p. 212).

Les conclusions tirées de ces différentes études plaident en faveur d'une formation des enseignants à la didactisation du brouillon, la qualité du texte final lui étant partiellement imputable. Mais, à nos yeux, ce type de formation n'a de sens que si les enseignants sont sensibilisés aux enjeux que représente un travail permettant aux élèves d'être les premiers auteurs-lecteurs-correcteurs efficaces de leurs brouillons, et de réhabiliter ainsi le statut de cet écrit. Ce travail de sensibilisation doit prendre en compte les représentations que se font les enseignants (ici algériens) du statut et de l'importance du brouillon et notre étude cherche donc à éliciter ces représentations.

\section{Méthodologie}

Pour évaluer les représentations des enseignants sur l'usage du brouillon à des fins didactiques auprès d'un public lycéen, nous avons dans un premier temps élaboré un questionnaire. Ce dernier a été envoyé à 40 enseignants de français, 34 l'ont complété et retourné (taux de réponse : $85 \%)$.

\section{Le questionnaire}

Le questionnaire adressé aux enseignants comporte 26 questions, équitablement réparties en questions fermées et ouvertes pour éviter les biais pouvant engendrer une liste de questions uniformes. Une logique dans leur enchainement est préservée afin de faciliter le cheminement du répondant. Celui-ci doit pouvoir suivre, plus ou moins, le raisonnement de l'enquêteur. Le questionnaire est divisé en quatre parties. La première permet de connaitre le profil du répondant ou de la répondante (données biographiques et professionnelles comme le sexe, l'âge, le diplôme de formation et le nombre d'années d'expérience). La deuxième partie vise à découvrir ce que les enseignants pensent du programme du français en lien avec la production écrite et à recueillir les opinions des enseignants sur le degré de réussite de leurs élèves, sur les causes de difficultés en production écrite et les solutions apportées. La 
troisième partie traite des pratiques d'enseignement de la production écrite, ses caractéristiques, ses priorités. Elle aborde directement les activités liées à l'apprentissage du processus rédactionnel et la question du brouillon. La quatrième et dernière partie est axée sur les pratiques évaluatives. Les questions abordent la proportion des textes corrigés avec suggestions ou commentaires écrits, le type de commentaires proposés, ainsi que la forme d'évaluation privilégiée, et les moyens mis en œuvre pour aider les élèves à s'auto-évaluer.

Les résultats que nous présentons ci-après concernent quelques questions qui étaient particulièrement destinées à cerner les représentations des enseignants quant à l'usage du brouillon et à recueillir leurs pratiques déclarées d'évaluation de l'écrit.

1. Sexe

3. Avec quel diplôme avez-vous été recruté dans l'enseignement secondaire?

4. En comptant cette année, combien d'années d'expérience en enseignement possédez-vous au total?

5. À combien de classes enseignez-vous le français?

6. Quel est le nombre moyen d'élèves dans vos classes de français, cette année?

10. Parmi les points suivants, quels sont ceux pour lesquels vos élèves éprouvent des difficultés? Mettez un numéro d'ordre de 1 à 6 .

11. Dans quelle mesure êtes-vous d'accord avec les causes des difficultés de vos élèves en expression écrite?

15. En classe, recommandez-vous à vos élèves de faire des brouillons avant de rédiger leur texte au propre?

16. D'après vous, environ quelle proportion de vos élèves font des brouillons pour leurs écrits en français?

20. Vous arrive-t-il de ramasser et de corriger les brouillons de vos élèves?

25. Prévoyez-vous des séances d'auto-évaluation ou de réécriture? 


\section{Synthèse des résultats}

Nous présentons ci-dessous les principaux renseignements de notre questionnaire.

\subsection{Les caractéristiques socioprofessionnelles des enseignants observés}

\begin{tabular}{|c|c|c|c|}
\hline Années d'expérience & Femmes & Hommes & Total \\
\hline Moins de 5 ans & 8 & 4 & 12 \\
De 6 à 10 ans & 0 & 0 & 0 \\
De 11 à 15 ans & 14 & 4 & 18 \\
De 16 à 20 ans & 0 & 4 & 4 \\
Plus de 21 ans & 0 & 0 & 0 \\
\hline Nombre total & 22 & 12 & 34 \\
\hline
\end{tabular}

Tableau 1. - Années d'expérience en enseignement de français selon les variables Sexe et Expérience.

Selon les données présentées dans le tableau ci-dessus, la population étudiée est constituée d'une majorité de femmes (65\%). 18 de ces enseignants ont entre 11-15 ans d'ancienneté dans le métier contre 12 seulement qui ont moins de cinq ans et 4 qui comptent 16 ans et plus. La moyenne se situe à 11 ans, on peut donc conclure qu'il s'agit d'un groupe d'enseignants expérimentés. Le croisement des données «sexe et années d'expérience» montre que la profession a tendance à se féminiser en Algérie comme ailleurs.

Tous les enseignants interrogés ont acquis un diplôme universitaire : 28 d'entre eux ont suivi une formation en licence de français, 6 répondent qu'ils ont suivi une formation bien différente de la nature du poste occupé dont 4 ont un diplôme d'ingénieur en biologie ${ }^{1}$.

1. Les biologistes et d'autres diplômés issus de filières scientifiques peuvent être recrutés en tant qu'enseignants de français dans les différents paliers du système éducatif algérien, à condition que leur formation universitaire ait été suivie exclusivement en langue française. Ainsi, nous sommes en droit de nous demander si l'utilisation qu'ils font du brouillon et la conception qu'ils ont de son utilité ne sont pas en lien avec leur formation d'origine : on ne fait pas un brouillon en production écrite en français comme on le fait en biologie ou dans une autre matière scientifique. 


\subsection{Les conditions du travail des enseignants observés}

\begin{tabular}{|l|c|c|}
\hline Q5 : Nombre de classes & Nombre & Pourcentage \\
\hline Trois classes & 0 & 0 \\
Quatre classes & 22 & $64,70 \%$ \\
Cinq classes ou plus & 12 & $35,29 \%$ \\
\hline Q6 : Taille moyenne d'une classe & Nombre & Pourcentage \\
\hline Moins de 25 élèves & 4 & $11,76 \%$ \\
De 26 à 40 élèves & 22 & $64,70 \%$ \\
40 élèves ou plus & 8 & $23,52 \%$ \\
\hline
\end{tabular}

Tableau 2. - Nombre et taille moyenne d'une classe.

La quasi-totalité des enquêtés enseigne à quatre classes. L'effectif des classes est globalement important, même s'il est variable : seuls 4 enseignants ont des classes de moins de 25 élèves, une large majorité des répondants (22 enseignants sur 34 ) enseigne dans des classes composées de 26 à 40 élèves et 8 dans des classes de 40 élèves ou plus.

\subsection{Les opinions des enseignants sur l'enseignement de la production écrite}

La question $\mathrm{n}^{\circ} 10$ demande aux répondants de classer par ordre croissant d'importance les points sur lesquels leurs élèves éprouvent des difficultés à l'écrit. 18 enseignants avancent les points suivants ( «rechercher et organiser les idées»; «orthographier correctement d'un point de vue lexical et grammatical»; «produire des phrases complètes et correctes»; «lier les éléments du texte en utilisant les connecteurs appropriés»; «relire et corriger le texte et enfin produire un texte d'une présentation matérielle acceptable»). Pour déterminer l'importance accordée à chaque difficulté, nous avons attribué des points pour chaque rang $\left(1^{\text {er }}\right.$ rang $=6$ points, $2^{\mathrm{e}}$ rang $=5$ points, $3^{\mathrm{e}}$ rang $=4$ points, $4^{\mathrm{e}}$ rang $=$ 3 points, $5^{\mathrm{e}}$ rang $=2$ points, $6^{\mathrm{e}}$ rang $=1$ point). Puis, nous avons calculé la moyenne qui se situe entre 1 au minimum et 6 au maximum. 


\begin{tabular}{|l|c|}
\hline $\begin{array}{l}\text { Q10 : Parmi les points suivants, quels sont ceux pour } \\
\text { lesquels vos élèves éprouvent des difficultés? } \\
\text { Mettez un numéro d'ordre de } \mathbf{1} \text { à 6. }\end{array}$ & \multirow{2}{*}{ Moyenne } \\
\hline Désignation & 5,35 \\
\hline Rechercher et organiser les idées. & 5,00 \\
\hline Produire des phrases complètes et correctes. & 4,58 \\
\hline Orthographier correctement d'un point de vue lexical et grammatical. & 3,94 \\
\hline Relire et corriger le texte. & 3,05 \\
\hline Lier les éléments du texte en utilisant les connecteurs appropriés. & 1,20 \\
\hline Produire un texte d'une présentation matérielle acceptable. & \\
\hline
\end{tabular}

Tableau 3. - Les difficultés des élèves à l'écrit selon les enseignants interrogés.

Pour la quasi-totalité des enseignants interrogés (30), «la recherche et l'organisation des idées» est la source de difficultés la plus importante chez les élèves. Selon ces répondants, c'est la conception du plan, l'organisation des idées, la mise en place des points de repères pour commencer à écrire qui constituent le premier blocage pour la plupart des jeunes scripteurs et non les problèmes de lexique.

Un des enseignants dit :

Quand je propose personnellement un plan à mes élèves, la plupart sont à l'aise et heureux. Ils commencent à rédiger, à exprimer leurs idées plus ou moins correctement. Nombreux sont, en effet, les élèves qui suivent à la lettre ce qu'on leur propose comme plan sans pouvoir modifier ou ajouter des idées.

La production de phrases complètes et «correctes» est la deuxième cause des difficultés évoquées par les enseignants interrogés. La mauvaise qualité des textes tient à la présence de phrases courtes, inachevées et impropres à la langue française. Ensuite, vient «l'orthographe correcte » qui est d'ailleurs la bête noire de la majorité des apprenants, même en langue maternelle. Enfin, «la relecture et la correction du texte» arrive au quatrième rang, suivi de «la liaison des éléments du texte». Des enseignants avancent que leurs élèves éprouvent des difficultés plus ou moins grandes pour relire et corriger leurs textes. Leurs interventions lors de cette relecture restent peu significatives et ne montrent pas de traces de réflexivité à propos du texte produit. En revanche, l'utilisation des connecteurs pour lier les différentes parties du texte pose moins de problème, les élèves du secondaire maitrisent 
cet aspect de la production écrite, compte tenu de leur confrontation régulière avec plusieurs textes de leur manuel qui sont «bien» structurés et liés. Par ailleurs, 22 des enseignants répondants trouvent que leurs élèves n'éprouvent pas de difficultés importantes pour «la présentation matérielle du texte».

\begin{tabular}{|c|c|c|c|c|c|c|c|c|}
\hline \multirow{2}{*}{$\begin{array}{l}\text { Q11 : Dans quelle mesure êtes-vous } \\
\text { d'accord avec les causes des difficultés } \\
\text { de vos élèves en expression écrite? }\end{array}$} & \multicolumn{2}{|c|}{$\begin{array}{c}\text { Pas du tout } \\
\text { d'accord }\end{array}$} & \multicolumn{2}{|c|}{$\begin{array}{c}\text { Plutôt pas } \\
\text { d'accord }\end{array}$} & \multicolumn{2}{|c|}{$\begin{array}{c}\text { Plutôt } \\
\text { d'accord }\end{array}$} & \multicolumn{2}{|c|}{$\begin{array}{l}\text { Tout à fait } \\
\text { d'accord }\end{array}$} \\
\hline & Nb & Fréq & $\mathbf{N b}$ & Fréq & $\mathbf{N b}$ & Fréq & $\mathbf{N b}$ & Fréq \\
\hline $\begin{array}{l}\text { Les élèves sont nombreux en classe pour } \\
\text { permettre un enseignement efficace. }\end{array}$ & 12 & $35,29 \%$ & 8 & $23,52 \%$ & 8 & $23,52 \%$ & 6 & $17,64 \%$ \\
\hline $\begin{array}{l}\text { Ils ont accumulé trop de lacunes au } \\
\text { cours de leur scolarité antérieure. }\end{array}$ & 0 & 0 & 6 & $17,64 \%$ & 10 & $29,41 \%$ & 18 & $52,94 \%$ \\
\hline $\begin{array}{l}\text { Ils évoluent dans un milieu social et } \\
\text { familial qui ne valorise pas l'écrit. }\end{array}$ & 4 & $11,76 \%$ & 6 & $17,64 \%$ & 14 & $41,17 \%$ & 10 & $29,41 \%$ \\
\hline $\begin{array}{l}\text { Ils manquent de confiance en eux et } \\
\text { n'imaginent pas qu'ils peuvent réussir. }\end{array}$ & 8 & $23,52 \%$ & 6 & $17,64 \%$ & 12 & $35,29 \%$ & 8 & $23,52 \%$ \\
\hline $\begin{array}{l}\text { Il y a un grand écart entre les objectifs } \\
\text { et les programmes d'enseignement de } \\
\text { l'écrit et les capacités des élèves. }\end{array}$ & 6 & $17,64 \%$ & 10 & $29,41 \%$ & 10 & $29,41 \%$ & 8 & $23,52 \%$ \\
\hline $\begin{array}{l}\text { Les élèves n'écrivent pas assez en } \\
\text { français. }\end{array}$ & 4 & $11,76 \%$ & 4 & $11,76 \%$ & 10 & $29,41 \%$ & 16 & $47,05 \%$ \\
\hline
\end{tabular}

Tableau 4. - L'origine des causes des difficultés des élèves selon les enseignants.

Moins de la moitié des enseignants interrogés (14) est tout à fait ou plutôt d'accord avec l'idée que le grand nombre d'élèves en classe est une cause principale des difficultés. Ainsi, la surcharge des classes n'est pas retenue comme une cause principale des difficultés à l'écrit par la majorité des répondants (20 enseignants). Ces derniers semblent ainsi considérer comme normal d'avoir des classes aussi importantes (réalité qu'ils ont d'ailleurs vécue eux-mêmes au cours de leur scolarité); il est vrai que ce phénomène s'intensifie dans le système éducatif algérien, même après la mise en place des réformes depuis l'indépendance. Parmi les dix autres causes proposées dans le questionnaire, la grande majorité des enseignants sont plutôt d'accord ou tout à fait d'accord avec les trois facteurs suivants :

1) le grand nombre de lacunes accumulées au cours de la scolarité antérieure $(82,35 \%)$; 
2) le peu de motivation pour l'écriture (les élèves n'écrivent pas assez) $(76,47 \%)$;

3) le manque de confiance (ils n'imaginent pas qu'ils peuvent réussir) $(70,58 \%)$.

Plusieurs enseignants imputent aux élèves la responsabilité de leur échec et avancent qu'ils arrivent au secondaire avec des lacunes telles qu'il faut d'abord reconstruire certains savoirs linguistiques et textuels. Pour toutes ces raisons, la rédaction parait souvent comme une activité lourde qui nécessite beaucoup de temps et d'efforts, comme en témoigne la réponse suivante : «Au lycée, les élèves sont faibles à l'écrit, c'est pourquoi, ils ne sont pas toujours enthousiastes à l'idée d'une production écrite; celle-ci est souvent associée à une corvée, aux fautes d'orthographes, aux ratures qui constituent encore une cause d'échec.» Un autre explique : "Les difficultés des élèves s'expriment par un blocage dû à la peur de faire des fautes d'orthographes. Les élèves écrivent comme ils parlent et ne se relisent pas souvent. Ce qui explique l'incohérence marquée dans leurs textes.»

Ainsi, les enseignants interrogés tendent à s'accorder sur le fait de ne pas imputer les raisons des difficultés de leurs élèves à l'écrit à la surcharge des classes mais à la non-maitrise de prérequis. Si les enseignants pointent des facteurs divers à la source des lacunes ou des faiblesses de leurs élèves, on remarque qu'aucun d'entre eux n'évoque cependant l'absence de tâche de réécriture, ce qui manifeste selon nous le caractère secondaire de ces activités dans les représentations des enseignants et sans doute dans leurs pratiques.

\subsection{Place accordée au brouillon en classe}

La question $\mathrm{n}^{\circ} 15$ «En classe, recommandez-vous à vos élèves de faire des brouillons avant de rédiger leur texte au propre?» avait pour but de vérifier si le brouillon faisait l'objet d'une pratique ordinaire et spontanée chez les élèves ou au contraire s'il résultait d'une contrainte imposée par leurs enseignants. Comme le montre le tableau 5 ci-dessous, 4 enseignants déclarent recommander très souvent aux élèves de faire un brouillon, contre 12 qui affirment le faire souvent, 10 qui l'exigent parfois et 8 qui ne le font jamais. 


\begin{tabular}{|c|c|}
\hline \multicolumn{2}{|c|}{$\begin{array}{l}\text { Q15 : En classe, recommandez-vous à vos élèves de faire des brouillons } \\
\text { avant de rédiger leur texte au «propre»? }\end{array}$} \\
\hline Désignation & Nombre d'enseignants \\
\hline Très souvent & 4 \\
\hline Souvent & 12 \\
\hline Parfois & 10 \\
\hline Jamais & 8 \\
\hline
\end{tabular}

Tableau 5. - Recommandation du brouillon par les enseignants.

Pour les enseignants qui estiment qu'il est important de faire un brouillon pour les écrits scolaires, nous retrouvons dans leurs justifications une partie des discours d'experts : le brouillon permet à l'élève de réfléchir soigneusement à la consigne, de récupérer toutes les informations nécessaires pour la rédaction, de se préparer à l'écriture au «propre». Les enseignants qui déclarent recommander parfois ou jamais de faire un brouillon avancent qu'ils préfèrent laisser plus de liberté à leurs élèves pour décider d'entrer ou de ne pas entrer dans une activité d'écriture déjà suffisamment ennuyeuse et lourde. Un enseignant biologiste dit : «Pour moi, je ne demande aucun brouillon, les élèves sont déjà conscients de la difficulté de la tâche qui nécessite beaucoup de temps. Pour eux, faire un brouillon est une perte de temps.»

La question $\mathrm{n}^{\circ} 16$ porte sur la proportion des élèves qui font un brouillon à leurs rédactions. Dans le cas où le brouillon n'est pas exigé personnellement par l'enseignant, c'est probablement qu'il serait un objet familier, fréquemment utilisé ou au contraire méconnu et négligé par les élèves. Ainsi, deux groupes de tailles égales apparaissent, comme le montre le tableau 6. Le premier groupe, constitué de 14 enseignants, évalue à $25 \%$ la proportion d'élèves de la classe qui font des brouillons. Le second groupe d'enseignants, qui comprend également 14 enseignants, estime que $50 \%$ des élèves en font. 6 enseignants seulement estiment que $75 \%$ de leurs élèves utilisent des brouillons pour leurs écrits en français. Cette différence d'appréciation par les enseignants peut s'expliquer par le fait que les élèves ont des pratiques diverses, en fonction de leurs propres habitudes et de leurs besoins. Elle reflète sans doute également le fait que le brouillon ne fait pas l'objet d'une exigence ou d'un enseignement explicite dans les classes de ces enseignants : si le travail sur le brouillon était davantage didactisé dans le cadre de l'enseignement de la production écrite, les chiffres obtenus à 
cette enquête auraient été plus homogènes et probablement supérieurs. Le croisement des réponses obtenues à cette question et celles qui la précèdent permet de confirmer l'idée selon laquelle le brouillon scolaire est en crise à l'école algérienne. Si les enseignants lui accordaient une place primordiale dans toute activité rédactionnelle, leurs élèves l'utiliseraient plus ou moins volontairement. Sa valeur se réduit selon les exigences des enseignants. Ainsi, réhabiliter la place accordée au brouillon, apprendre aux élèves explicitement son enjeu est une nécessité urgente en classe du FLE.

\begin{tabular}{|c|c|}
\hline $\begin{array}{l}\text { Q16 : D'après vous, environ quelle proportion de vos élèves font des } \\
\text { brouillons pour leur écrits en français? }\end{array}$ \\
\hline Désignation & Nombre d'enseignants \\
\hline $25 \%$ & 14 \\
\hline $50 \%$ & 14 \\
\hline $75 \%$ & 6 \\
\hline $100 \%$ & 0 \\
\hline
\end{tabular}

Tableau 6. - Proportion des élèves qui font un brouillon d'après les enseignants interrogés.

\section{5. Évaluation de la production écrite}

Nous voulons à travers la question $\mathrm{n}^{\circ} 20$ vérifier le statut du brouillon dans les pratiques évaluatives des enseignants : l'introduisent-ils comme objet didactique évaluable ou le prennent-ils comme pis-aller de la production des élèves? Le tableau 7 montre que 14 des enseignants interrogés ramassent souvent le brouillon de leurs élèves mais ne le font que parce que les élèves ne finissent pas la rédaction de leur texte au «propre», ce qui oblige alors les enseignants à prendre leur brouillon comme trace écrite de leur production. 12 enseignants reconnaissent qu'ils ne ramassent et ne corrigent jamais le brouillon de leurs élèves. Ces réponses montrent que le brouillon n'a pas réellement de place dans les pratiques évaluatives des enseignants et confirment ainsi l'analyse de la question précédente. 


\begin{tabular}{|c|c|}
\hline Q20 : Vous arrive-t-il de ramasser et de corriger les brouillons de vos élèves? \\
\hline Désignation & Nombre d'enseignants \\
\hline Très souvent & 0 \\
\hline Souvent & 14 \\
\hline Parfois & 8 \\
\hline Jamais & 12 \\
\hline
\end{tabular}

Tableau 7. - Correction du brouillon.

La question $\mathrm{n}^{\circ} 25$ cherche à savoir si les enseignants impliquent leurs élèves dans d'autres modes d'évaluation, à savoir l'auto-évaluation, liée particulièrement à la relecture des premiers jets.

\begin{tabular}{|c|c|}
\hline Q25 : Prévoyez-vous des séances d'auto-évaluation ou de réécriture ? \\
\hline Désignation & Nombre d'enseignants \\
\hline Très souvent & 6 \\
\hline Souvent & 14 \\
\hline Parfois & 10 \\
\hline Jamais & 4 \\
\hline
\end{tabular}

Tableau 8. - Programmation des séances d'auto-évaluation.

Les résultats montrent que 20 des répondants déclarent avoir très souvent ou souvent l'occasion de prévoir des séances d'auto-évaluation et avancent que cette technique permet à l'élève de faire un bilan personnel de son progrès. En outre, selon ces enseignants, cette procédure d'évaluation étant recommandée par l'institution, ils la pratiquent une fois par séquence, comme en témoigne l'extrait d'entretien suivant :

Dans le nouveau programme, on doit programmer des séances d'autoévaluation au moins une fois à l'issue de chaque séquence, les élèves écrivent leurs premiers jets, puis l'enseignant les corrige, les annote et les leur remet encore une fois pour qu'ils corrigent leurs erreurs et améliorent leurs textes.

10 enseignants disent qu'ils prévoient parfois des séances d'autoévaluation et expliquent d'une part que le temps alloué à l'enseignement de la production écrite n'est pas suffisant pour programmer des séances de réécriture et d'autre part, que la surcharge des classes ne favorise pas la mise en place de cette activité. Rappelons que cette dernière raison a été avancée par environ 14 enseignants pour justifier les 
difficultés de leurs élèves en situation de production écrite. Toutefois, ils estiment qu'il est nécessaire d'amener les élèves à relire et à évaluer leur production écrite et à juger par eux-mêmes leur niveau de difficultés, comme le montre ce témoignage : «Personnellement, je fais parfois ce type d'activité pour l'ensemble de la classe, mais pour la réécriture, je sélectionne quelques copies seulement car j'ai des classes nombreuses.»

4 enseignants seulement reconnaissent n'avoir pas l'occasion de programmer des séances d'auto-évaluation. Ainsi, nous pouvons dire que même si l'auto-évaluation est une injonction forte de l'institution, les modalités pédagogiques sont encore peu développées pour la pratiquer.

\section{Conclusion}

Cette étude visait à décrire la place accordée au brouillon dans les pratiques évaluatives des enseignants du secondaire et les conceptions de l'enseignement et de l'évaluation de la production écrite. Elle fait apparaitre que près de la moitié des 34 enseignants interrogés recommande aux élèves de faire un brouillon pour leur production écrite. Selon leurs déclarations, le passage au brouillon permet à l'élève de réfléchir soigneusement à la consigne, de récupérer toutes les informations nécessaires pour la rédaction, de se préparer à l'écriture au «propre». Cependant, la plupart des répondants n'accordent pas un grand intérêt au brouillon qui ne fait pas l'objet de rétroactions écrites : leurs annotations portent sur le texte final et restent peu nombreuses, ce qu'ils justifient par le souci de ne pas démotiver les élèves.

Nous pouvons affirmer que le brouillon est encore loin d'être didactisé à l'école algérienne. Il parait donc essentiel de poursuivre des recherches pour comprendre comment aider les enseignants à intégrer la pratique du brouillon comme outil didactique supplémentaire permettant progressivement aux élèves d'améliorer leurs écrits par le développement de leur regard réflexif sur la pratique de l'écriture comme processus et non seulement comme produit. Notre recherche doctorale (Benabbes, en préparation) montre que la correction des brouillons par les enseignants, amenant l'élève à un travail guidé de réécriture, est plus efficace que l'activité de révision/correction menée par l'élève luimême via l'utilisation d'une grille d'auto-évaluation. La multiplication de ces travaux et leur exploitation en formation d'enseignants permettront sans doute d'améliorer la qualité pédagogique de l'enseignement de la production écrite du FLE dans le système scolaire algérien. 


\section{RÉFÉRENCES BIBLIOGRAPHIQUES}

AlCORTA, Martine. (2001). Utilisation du brouillon et développement des capacités d'écrit. Revue française de pédagogie, 137, 95-103.

Bellemin-NoËL, Jean. (1977). Reproduire le manuscrit, présenter les brouillons, établir un avant-texte. Littérature, 28, 3-18.

Benabbes, Souad. (En préparation). Accompagner les élèves de $3^{e}$ année secondaire pour une amélioration de la pratique scripturale en FLE (Thèse de doctorat en didactique, Université de Batna).

Brown, James. (2007). Feedback: The Student Perspective. Research in Post-Compulsory Education, 12(1), 33-51.

CARTER, Michael. (1990). The Idea of Expertise: An Exploration of Cognitive and Social Dimensions of Writing. College Composition and Communication, 41, 265-286.

CuQ, Jean-Pierre (dir). (2003). Dictionnaire de didactique du français langue étrangère et seconde. Paris : CIE international.

Dembri, Naima. (2008). L'usage du brouillon dans l'acquisition du savoirécrire (Mémoire de magister en didactique, Université de Constantine). Disponible en ligne sur $<\mathrm{http} / / /$ bu.umc.edu.dz/theses/francais/DEM10 53.pdf> (consulté le 28 mars 2016).

Dore, Robert. (1981). Influence relative de modalités de rétroaction dans l'apprentissage chez des enfants déficients mentaux moyens (Thèse de doctorat). Université de Montréal.

FABRE, Claudine. (1987). Les Activités métalinguistiques dans les écrits scolaires (Thèse de doctorat d'État). Université Descartes - Paris 5.

FABRE-Cols, Claudine. (2002). Réécrire à l'école et au collège. De l'analyse des brouillons à l'écriture accompagnée. Paris : ESF.

Hassan Mohamed, Rania. (2011). Les croyances des enseignants et des apprenants adultes quant à la rétroaction corrective à l'oral et la pratique réelle en classe de français langue étrangère en Égypte (Thèse de doctorat). Université de Montréal.

Flower, Linda \& HAYES, John R. (1980). Identifying the Organization of Writing Processes. Dans L.W. Gregg \& E.R. Steinberg (dir.), Cognitive Processes in Writing (p. 31-50). Hillsdale, NJ : Erlbaum.

Ministère de l'Éduchtion nationale d'Algérie. (2006). Document d'accompagnement du programme de français de $3^{e}$ année secondaire.

Penloup, Marie-Claude. (1994). «La Rature n'est pas un raté». Plaidoyer pour le brouillon. Rouen : MAFPEN.

Peterson, Stagg. (2010). Faire la différence... De la recherche à la pratique. Monographie, 29, 1-4. Disponible en ligne sur <www.edu.gov. on.ca/fre/literacynumeracy/inspire/research/WW_Student_Writing French.pdf>. 
Piolat Annie, Roussey, Jean-Yves \& Fleury, Philippe. (1994). Brouillons d'étudiants en situation d'examen. Le français aujourd'hui, 108, 39-49. Reuter, Yves. (2002). Enseigner et apprendre à écrire. Paris : ESF.

RoDET, Jacques. (2000). La rétroaction, support d'apprentissage? Revue du conseil québécois de la formation à distance, 4(2), 45-74. 